\title{
SOFTWARE RADIO: EVOLUTION OR REVOLUTION IN SPECTRUM MANAGEMENT
}

\author{
Rebecca Cowen-Hirsch \\ Deputy Director, DISA Office of Spectrum Analysis and Management (OSAM)
}

\author{
Dick Shrum, Brian Davis, Dennis Stewart, Kalle Kontson \\ IIT Research Institute
}

\begin{abstract}
Leveraging new technologies and developing new approaches to enable more effective and efficient use of the electromagnetic (EM) spectrum are central to achieving the Information Superiority that is key to the emerging operational concepts described in Joint Vision 2010. Fortuitously, evolving software radio technologies will lead to the emergence of adaptive, "self-managing" systems that offer the potential for quantum improvements in spectrum efficiency through real-time, dynamic spectrum reuse. Central to these "smart" systems will be the control of radio functions by embedded software algorithms, directly affecting the manner in which the system uses the EM spectrum. However, positive control over a radio's use of the spectrum has traditionally been the purview of radio operators, who were guided and trained to follow the rules of engagement carefully crafted over decades to satisfy mission requirements while preventing harmful interference to other users. This power of control is now being handed over to the radio itself. The obvious question is: How does this transfer of control affect the spectrum management policies, practices, and procedures devised to support, guide, and manage the behavior of human operators? This paper addresses this central question as it relates to Spectrum Management business practices.
\end{abstract}

\section{THE MILIT ARY REQUIREMENT}

The battlefield of tomorrow is well described in DoD's Joint Vision 2010. It provides the conceptual template for leveraging technological opportunities to achieve new levels of effectiveness in joint warfighting for a challenging and uncertain future. The vision develops four operational concepts: dominant maneuver, precision engagement, full dimensional protection, and focused logistics. More important to this subject, it also cites the unqualified importance of information in the future, and assumes an improved level of intelligence, command and control befitting of today's information age. Information Superiority-the capability to collect, process, and disseminate an uninterrupted flow of information while exploiting or denying an adversary's ability to do the same-is the critical ENABLER of Joint Vision 2010.
Achieving and sustaining Information Superiority, and success on the battlefield, require the use of advanced technology in future military communications-electronics (C-E) systems. Military planning, development and procurement include a broad range of new technologies, such as adaptive, self-managed, versatile radios, to satisfy a global requirement for "anywhere, anytime" capability. There is a critical need to maximize and manage information flow on the battlefield, which in turn requires system interoperability among services, battle elements, and allies. These C-E systems must operate with relatively unconstrained freedom in sufficient radio frequency bands. To this end, DoD has developed Joint Spectrum Vision 2010 , which provides a coherent view of future spectrum implications and a guide for pursuing specific initiatives. Assured spectrum access is the ENABLER of Information Superiority.

\section{WIRELESS EXPLOSION AND "SMART RADIO"}

Advances in semiconductor technologies have yielded ever increasing processor speeds and addressable memory in ever smaller packages. These developments, combined with burgeoning demand for new wireless applications and services, have resulted in the explosive growth of digital radio technologies. Software programmable, high speed digital signal processors are common and "smart radio", as realized through software control of radio functions, is here today and available off-the-shelf. The unlicensed Bluetooth devices, providing a radio link in the $2.4 \mathrm{GHz}$ band to replace connection cables to printers, keyboards and Personal Digital Assistants (PDA), reflect the leading edge. The Bluetooth system specification is all-inclusive, persistent, fully automated, and self-managing, and it should contribute directly to the development of future highly adaptive, flexible and interoperable radio systems.

In this light, two categories of "smart radios" are defined for context as they relate to the progressing evolution of the technology. "Software Defined Radio (SDR)",[1] is best characterized as a device in which most signal processing is performed in the digital domain using programmable Digital Signal Processors (DSP) and hardware support, with some processing still done in the analog domain, such as RF and UHF and above IF circuits. "Software Radio 
$(S W R)^{\prime[2]}$ reflects the fully robust implementation of radio functionality in software. In this device, the antenna is connected directly to an analog-to-digital (A-D) converter and signal processing is all digital using fully programmable high speed DSPs, and functions, modes, applications, etc. can be added, configured, and upgraded by software. Existing technology can easily produce some SDR devices immediately, with the development of a fullblown SWR device possible in the not-too-distant future. Indeed, as processor speeds increase beyond the current 1 $\mathrm{GHz}$, no down-conversion will be required to process incoming RF signals, and digitization will occur at source frequencies, thus expanding the realm of software controlled radio functions.

Software radio offers a unique and powerful solution for wireless systems, in that it is neutral to the variety of standards and other specifications in use or under development ${ }^{[3]}$. It provides a "future proof" architecture for the evolution of adaptive and interoperable wireless, the capability to reconfigure an RF network-quickly, cheaply and with great flexibility, and an effective vehicle to merge wireless telephony and data communication with computing capability. SDR technology plays a major role in the complex task of network management, and is capable of flexible adaptation to network protocols, billing and accountability requirements, as well as technical parameter selection and control. Today's SDR will progress into SWR in the future, as technologies and requirements evolve. SWR will be a remotely reprogrammable device analogous to the networked PC, capable of accepting radio and network control algorithm updates in the same way that new applications can now be downloaded from the Internet. As for the computer, global harmonization will likely occur in software architectures and approaches, since they will control how the device interacts with its host medium - the radio frequency spectrum.

DoD views software radio as a critical technology to satisfy a global requirement for interoperability among the services and with allies, as well as a means to achieve information superiority, operational flexibility and associated cost-reduction benefits. Its plan is to proceed with the rapid introduction of SDR in the near term, transitioning to a full-blown, ultra-smart SWR later. The Joint Tactical Radio System (JTRS) Program ${ }^{[4]}$ portends a family of advanced, software controlled and reprogrammable radio platforms, capable of multiband, multimode, multi-bandwidth operations. They will incorporate new waveforms and technologies; network with simultaneous voice, data, and video; be highly mobile - air, ground, sea, "anywhere/anytime"; and interoperate with military and civilian legacy equipment. The Navy
Digital Modular Radio (DMR) replaces many incompatible radios, reducing operating costs and size/weight requirements, and enables the user to change bandwidth, modulation, security and waveforms with software commands, rather than the existing more costly hardware approach. JTRS and DMR represent the new breed of military software radio.

Implementation of software radio in military and commercial wireless systems will enable many benefits, ranging from more efficient technical and operating characteristics to expanded services including broadband and multimedia, from reduced procurement and operating costs to more flexible, adaptive and interoperable communications. More importantly, the most far-reaching benefit of software radio relates to its potential for making far more efficient use of the spectrum than the current processes allow. By viewing the RF medium as a host for digital radio "packets", multiple users and functions can be accommodated and managed, on a time-shared packet-bypacket basis similar to the Internet Protocol (IP) mechanism and use the entire information capacity of the spectrum resource. When combined with the ability to monitor the RF environment and its location, software radio opens the possibility of sharing spectrum among vastly different users and choosing compatible shared frequencies to avoid interference.

For example, imagine a "super-system" radio architecture in which all radiating devices "chat" with each other according to some agreed protocol, so that each device knows where its RF siblings are, what their needs are, and how best to coordinate use of the shared resource in time, location, frequency, and so forth. This would be the ultimate air-interface, coordinating spectrum usage far more rapidly and effectively than our operator-based process would allow. If this seems too far-fetched, review the evolution of PC's and, for example, the Bluetooth technology. Few envisioned video and audio broadcasting, point-to-point telephony, data exchange, email, high quality music, streaming video, facsimile, printing, video games, and what have you-ubiquitous and wireless-supported by one shared, seemingly chaotic infrastructure. However, it is here, and it will only get bigger and better.

\section{SPECTRUM MANAGEMENT IMPLICATIONS}

Existing spectrum policy, rules and approaches are not now designed to adequately handle SDR and SWR, let alone take advantage of their potential benefits. Work is underway to understand and develop the best model for spectrum access, technical standards and operating etiquette. For example, the frequency usage principle of 
"first-come, first-serve" is not applicable to an adaptive highly-shared environment, and it might be replaced with a "y'all come" philosophy. In addition, certification methods for hard-wired radio circuits are not applicable to situations where equipment configuration changes are performed in the field through software download. For the regulatory authority, control of radio functions directly affects the manner in which that device uses the spectrum. In the past, positive control of the transmitter was the purview of trained radio operators who could prevent harmful interference to other users, but now this power to control is being handed over to the device itself.

For spectrum management purposes, it is useful to distinguish between SDR and SWR, since they have different characteristics, complexity, implementation timeframes, and degrees of departure from current practices. However, they both offer the potential for increased spectrum use efficiency, and a solution to recapture lightly used or unused bands and channels, accommodate more users in a given band, and increase sharing. Early SDR will operate much like legacy devices, using the same bands, channel plans, standards, technical restrictions, operating procedures, and network protocols. They can use the same service definitions, rules, and methods to access the spectrum. The frequency assignment will be about the same as for a legacy radio, except it may contain several parts, one for each band/standard combination used. Hence, the first generation SDR system may seem indistinguishable from today's cellular, PCS or other operation, simply using its software to perform tasks more quickly and efficiently than the human operator ever could.

What is new and different, even with the first SDR, is the use of software to generate a variety of waveforms depending on the user's location, choice of media and its local standard, plus the ability to add or download new or upgraded versions of the software package. SDR devices can be pre-programmed to ensure compliance with regulations, standards and network factors, similar to the way present radios are pre-wired to give a desired result, and able to decide which power to use to achieve a desired service quality. How will a regulatory authority ensure that the "software" is correct initially and not modified later without authority to do so (hardware is easy to test and inspect)? If SDR can download new user services (e.g., add a broadband capability for Internet access) or another band/standard combination to allow greater roaming, how does the regulator (or regulators, if a second country is involved) ensure compliance with applicable regulations and avoidance of unforeseen interference from the new software?
Software Radio (SWR), on the other hand, reflects dynamic software-defined capability throughout the device, including all RF functions. It can be reconfigured by software download (like today's use of the Internet from a PC), possibly using an over-the-air transmission from the manufacturer, service operator, regulator, or a relatively unskilled third-party software vendor. The radio will adapt to its environment, changing bands or service providers as desired, selecting the necessary mode/waveform to respond to the user's request or an incoming call, monitoring a channel before transmitting to avoid interference to others, and even raising power to ensure communication in an emergency. With appropriate software, SWR can transmit anywhere, anytime, on any frequency with any mode or modulation desired, thus providing the military a powerful communications capability that enables Information Superiority and battlefield dominance. It is less clear how the private sector will use the full-blown software radio, but SWR can easily provide the range of services in the most effective, economical and flexible fashion. But for both groups, software will automatically control spectrum sharing and efficiency, avoid interference to other users, handle billing information for commercial services, perform record keeping for "pay as you go" and "spot market" spectrum access approaches, download new or improved applications as they are released, and arrange for customized services to the individual user.

In this light, SWR will diverge much more from existing policies and rules, requiring major changes to spectrum management philosophy and practice. For example, early software radios may derive their RF characteristics from standardized software modules and hardware platforms that can be handled using a "legacy concept" authorization procedure to verify performance of the original configuration of hardware, firmware and software components. Field upgrades and "on-the-fly" additions will require much more flexible and innovative acceptance testing of the software and careful management of the new configuration, maybe using a verification or declaration of conformity-type procedure done by the manufacturer, user or other responsible party. As access is granted to more bands and services, traditional concepts of allocation, service, assignment, and so forth will become less relevant. In view of the highly integrated and interactive nature of the system's components, it may be best to rely on industry and users, working jointly through associations or coordinating bodies, to develop software standards and publish guidelines for operation, configuration, version maintenance and field updating. If SWR is as good as some predict and can reliably manage its own use of the spectrum, then the role of regulators in the long term might 
reduce to publishing a short list of prohibited bands to protect historical services.

\section{MANAGEMENT MODELS AND PRACTICES}

Over the years, the spectrum authority or regulator has developed several models for managing the radio spectrum and its diverse users, taking into account various factors such as the state of technology, engineering capability, importance (e.g., safety of life), service quality, and sharing considerations. Three are useful for discussion purposes in this paper, as follows.

"Rigid rules" imply highly restricted bands, detailed technical/operational rules, tightly controlled usage by number and entrant, and virtually no room for flexibility (e.g., aviation/marine services/bands, broadcast, and radionavigation). A major disadvantage is that channels in some services (not applicable to continuous transmitters, such as broadcasting) are lightly used or unused.

"Flexible sharing" promotes sharing as a policy objective, has many users per channel operating under blanket license, regulates basic framework/technical details (e.g., power, channels, modulation) but leaves operation mainly to system licensee, and may involve an operating etiquette such as "listen before talking" or tone coded squelch (examples include PCS, trunked systems, amateur service, and private radio).

"Unrestricted" implies minimal rules (e.g., power/field strength limits, band restrictions) and relatively unlimited flexibility in application and area of use, subject to a strict "no protection given/no interference caused" provision. There are up to millions of users per channel, operating unlicensed PCS, garage door openers, cordless telephones, and many other FCC Part 15 devices.

In the early days of radio, management was primarily of the rigid type to establish order out of chaos and ensure an orderly development of radio systems. Even now, some radio applications still need a high level of specificity and structure, such as over-the-air broadcasting, aviation, maritime, and safety of life. As technology advanced, experience grew, and more users had to be accommodated, improved models - such as for cellular networks and unlicensed services-were adopted and implemented.

Thus in considering a new technology or radio service, the regulator considers and adopts rules for the various "business practices" of the spectrum management process. This effort can also be viewed from the perspective of establishing rules to optimize the "spectrum trade-space" in direct response to requirements and objectives, while protecting other services. Optimization of this trade-space refers to the prescription of RF parameters (such as frequency band, bandwidth, power, modulation, access method, antenna characteristics, and spectrum efficiency) to meet radio service requirements (such as coverage, data speed, message duration, number of simultaneous users, access priority, Quality of Service, etc.) As technology evolved from fixed frequency/bandwidth analog systems toward today's digital techniques, spectrum management moved from the "rigid rules" model to those of "flexible sharing" and "unrestricted" because the latter support innovation and variation of RF parameters to better accommodate user needs, service objectives and marketplace conditions. It is in this light that we should approach the challenges of software radio; specifically, the technology allows us to optimize and dynamically manage this spectrum trade-space, in real-time, to support spontaneous diversity in user services. The regulator's challenge is to adopt models and business practices that support this real-time optimization.

This paper looks at three aspects of the several spectrum management practices needed for software radio communications devices. The first is access to the spectrum. The traditional approach involves classification as to service type (e.g., fixed, mobile, government/nongovernment, trunked, maritime), an allocation reserving a designated band or channels for a particular user group/purpose, transmitter operating authority in the form of a license or assignment that specifies the channel(s) approved for use. Assignments may be granted via a firstcome, first-serve application process, by auction, or on a contention basis. Contention covers unlicensed devices or licensed services such as amateur radio in which an available frequency is selected from an allotment of authorized channels. Within the authorized frequency pool, an individual channel may be selected because it is at the moment free of other users or otherwise most optimal at that instant for the intended communication.

The success and efficiency with which unlicensed devices have been able to utilize the 915 and $2450 \mathrm{MHz}$ ISM bands hints at an updated spectrum access approach suitable for advanced SDR waveform applications. Unlicensed devices incorporate robust waveforms allowing them to share spectrum with other users. This ad hoc sharing is achieved without the infrastructure required by shared access schemes such as trunked radio services, cellular, and PCS. Spectrum requirements for systems assigned a single frequency per net could be compressed by applying a similar type of dynamic ad hoc spectrum access approach. SDRs would be the ideal type of device for this purpose because they contain processors capable of making reliable dynamic spectrum access decisions. 
The potential of the technology suggests that it need not be constrained to narrow service types, given its capability to handle various standards, waveforms, configurations, protocols, and operating etiquettes. It could, for example, access FCC-licensed commercial, Government trunked, maritime and public safety systems in a 15 -minute period, followed by a wideband videoconference carried out in a temporarily unused military radar band. While the service type and allocation concepts may be manageable albeit cumbersome, the serious inadequacies relate to operating authority and spectrum access. Software radio offers many innovative possibilities, such as its ability to use identifiable data packet bursts that could enable it to operate on another user's channel without interference, or to negotiate and carry out "spectrum trading" or "pay-asyou-go" transactions for each burst sent. Current rules make no provision for these possibilities, and rule updates will be needed to make the spectrum access protocol a major criteria in the assignment process.

A second aspect of new spectrum rules involves technical standards and operating etiquette, usually needed in the form of conditions or limitations to avoid interference to other spectrum users, to ensure viability of the authorized service(s), and in limited cases to provide a desired quality of service. Spectrum management technical standards traditionally have governed parameters such as maximum RF output, RF bandwidth, modulation, spurious emission levels, receiver selectivity, and frequency channel spacing. Future standards may include some of these traditional requirements in addition to requirements governing spectrum access etiquette. An indication of this trend is the operating etiquette for IEEE 802.11 wireless LAN devices in section 15.247 of the FCC rules. Complex waveforms spanning wide frequency ranges will require the ability to adapt their spectrum access etiquette to the unique requirements of multiple bands. A "Spectrum Control" object might perform this function as in Figure 1.

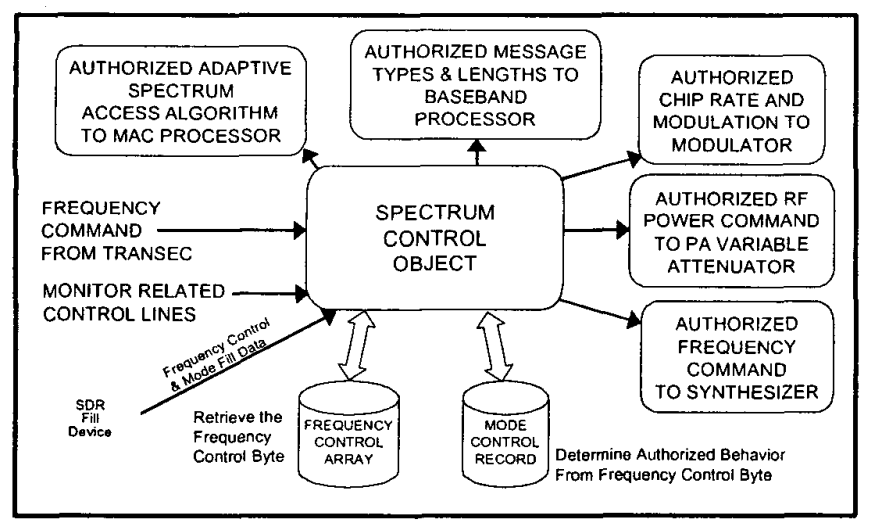

Figure 1. Spectrum Control Concept.
In this approach the Frequency Control Array contains a pointer to a mode description contained in the database of mode control records. This enables the Spectrum Control object to tailor the use of each authorized frequency to appropriate limits for power, bandwidth, duty cycle, and access etiquette. The etiquette would specify other conditions that must be met for the channel to be suitable. For example, a condition might be that the sensed ambient signal level must be below a specified level. Another possibility could be that communications need to be synchronized with the scan rate of cochannel radars. In some bands a coordination channel might exist and transmissions could not proceed until the control channel is polled for an appropriate response. This type of dynamic spectrum access and control can only be workable if the control algorithms and etiquette's are standardized via the equipment certification process.

The third aspect of management practices is certification and equipment approval. This is the formal process of testing a radio transmitter for compliance with applicable rules and approving its use for particular purposes, services and bands. Until now, this procedure involved equipment using hardware, sometimes with nonprogrammable firmware and related digital technology, that could be assumed to remain as originally inspected until someone opened the box and made changes. The incorporation of software, with the possibility of upgrades and downloads, is dramatically different for the regulator, and changes the entire nature of the approval process. For example, the DoD JTRS likely portends several new and unique features that will have to be addressed, such as:

- Mix of hardware, firmware, software to operate the radio

- Control software to manage and perform radio functions

- Ensuring that software implementations of legacy waveforms have RF characteristics meeting or exceeding those of legacy systems

- Advanced adaptive techniques too complex to evaluate and test manually

There is growing recognition that certification and equipment approval procedures, including the handling of subsequent upgrades and additions, should be developed in conjunction with system architectures and protocols, probably by the appropriate industry organization since they may be the only body that can fully understand the technology and its implemented systems. Another distinction of future regulatory procedures compared to existing rules will be a significantly increased reliance on automated tools for certification, verification and enforcement purposes. 


\section{VIEW FROM THE FCC}

In March 2000, the Federal Communications Commission issued a Notice of Inquiry ${ }^{[5]}$ regarding software defined radio requesting advice on the current state of technology and whether changes to $\mathrm{FCC}$ rules are necessary to accommodate SDR. It is clear from the Notice that FCC staff is confident about the benefits of the technology and its potential to improve the efficiency of spectrum usage and overcome existing incompatibilities. The FCC seeks comments in five specific areas: the state of technology, interoperability issues, spectrum efficiency issues, equipment authorization processes, and other relevant issues, as illustrated by the following questions.

- What are the commercial uses for SDR and the radio features likely to be controlled by software?

- Can SDR improve interoperability among public safety agencies, and between equipment and services using differing transmission standards?

- How much can SDR improve the efficiency of spectrum usage and spectrum sharing, and how would it perform this role?

- Should FCC approve radio hardware, software or both, and how should the SDR be tested for compliance? How should FCC handle anti-tampering, authentication, privacy, and other security requirements?

- Are there other issues to be considered, such as enforcement and accessibility for the disabled?

The Commission intends to move forward with the next step of the proceeding - a Notice of Proposed Rulemaking (NPRM) - in early 2001, followed in due course by appropriate rules and regulations. (Note by the author: It is intended that a progress report on this proceeding will be part of a presentation at MILCOM 2000.)

\section{FUTURE SPECTRUM APPROACHES}

Spectrum management policy and rules for software radio devices will be a combination of evolution \& revolution. First generation SDR equipment is fully capable of and should be expected to comply with the individual operating and technical requirements of each service/band combination used, whether hardware, firmware or software handles the function. It can readily operate under any of the traditional models described above, and there does not appear to be any substantial handicap, loss of performance, or inefficiency in so doing. Nevertheless, experience gained with early SDR systems will provide invaluable insight into how policies and rules should evolve to get maximum benefit from the technology. No major problems are foreseen for most of the spectrum management business practices; that is, there is no immediate need for new allocations, service definitions or categories, assignment methods, technical rules, since SDR will operate much like a legacy transmitter. However, the area of certification and equipment approval requires new or modified procedures particularly for software components and the ability to upgrade or modify in the field.

Advanced SDR/SWR equipment should have minimal operating and technical rules to allow maximum flexibility and innovation in implementing potential benefits. For example, this might include provisions for no operation in certain safety/passive bands, "no harm" to legacy systems/stations, no protection from interference (since SWR can adapt), and if necessary some reasonable power limitations. SWR may require a family of band-by-band rules (e.g., spread spectrum permitted in a given band only if a pre-designated minimum transmission bandwidth is observed), particularly at the start, with a view to reducing such rules as experience is gained and confidence is earned. Rules must take into account a transition period when autonomous SWR systems must coexist with more traditional "legacy" systems, recognizing that the latter are not as nimble and adaptive as SWR and extra care must be taken to ensure they do not become victims. However, the problem should not be viewed as insolvable since accommodating this mix of new and old technology correlates with challenges addressed as a result of recent radio service spectrum "refarming" actions. Further, some band/service combinations experience a relatively rapid changeover of equipment, which may allow for a more aggressive implementation of new rules and less pressure for lengthy "grand-fathering" periods. The greatest challenge will probably involve the rules needed to optimize the benefits of efficient spectrum usage and improved sharing, since they could reduce some of the flexibility desired for operational and technical purposes.

DoD must stay alert for the military-unique considerations of software radio. The military vision is focused on communications and information requirements ten years and more into the future, and already projects heavy reliance on advanced SWR technology, whereas the private sector will focus on short/mid-term issues of commercial systems such as multi-band/standard units to merge the $1 \mathrm{G} / 2 \mathrm{G}$ systems and to implement $3 \mathrm{G}$ technology when the market is ready. Hence, DoD should not rely too heavily on industry to carry the ball on spectrum management rules, but be ready to take the initiative where appropriate, particularly concerning longterm spectrum policy for advanced software radio systems. 
AN INNOVATIVE FUTURE SPECTRUM MODEL Software radio may be the enabling technology to exploit the advantages of an "Open Spectrum Access" wherein spectrum is viewed as a public right of way without specific ownership of bands or channels, and access rights would be granted by some effective and equitable market-specific means. It is important to note that this is not an expanded Part 15 approach, where only equipment manufacturing is regulated and day-to-day access is not managed or enforced. Open access means that once granted access rights, a user joins the multitude of shared users in accessing a common band according to a universal air-interface protocol that observes strict accountability, traceability and enforcement as an integral part of that protocol. It shifts the economic valuation of spectrum from the concept of "channels owned" to a more fundamental commodity; that is, "information capacity used" and "pay as you go". Open spectrum access might also be implemented using the concept of "spot markets" as envisioned by FCC Chairman Kennard ${ }^{[7]}$, or even the "Guard Band Managers" concept recently adopted by the FCC in part of the $700 \mathrm{MHz}$ band. It has been proposed to conduct a demonstration in an appropriate band, possibly under an experimental license, and that a new "Part X" in the FCC Rules be developed and adopted to implement this approach.

\section{THE CENTRAL QUESTION}

Whatever the model and rules adopted for software radio, the single most significant and prevailing implication of the technology is the concept of replacing the traditional "radio operator" with software! How does this transfer of control affect spectrum management policies, practices, and procedures devised to support, guide and manage the behavior of human operators? This is a valid question and the details remain to be hammered out, but there is precedence from the past. One example goes back nearly a century to the Titanic disaster, which led to the treatybased adoption of Morse code, SOS and the "shipboard radio operator". When this highly paid individual and outdated technology was proposed to be phased out of the maritime mobile service, in favor of automated direct printing and reliable voice systems, there was major resistance from radio unions and dire forecasts of lost lives and sunken ships. To nobody's surprise, there was a smooth and safe transition with substantial cost savings and other efficiencies when the change was finally made. In another example, we witness on an almost daily basis the "evolving revolution" of the computer. In the very early days, the original highly-trained "computer operator" ran mainframe systems, loading boxes of computer punch cards and returning reams of computer print-out paper for individual users. This "indispensable expert" disappeared almost overnight, as industry evolved to powerful desktopbased and user-operated computers, with greatly improved efficiency, effectiveness, flexibility, and a long list of other benefits. We somehow lived through the transition, to marvel at the benefits given to us by the new technology.

There are important questions about a paradigm shift involving software radio and its powerful capabilities. Can the software tools already being installed in wireless devices to provide multiband/multimode capability, security and a full range of services including Internet access, as well as sophisticated network control and management, be harnessed to also provide consistent and reliable control of the radio characteristics of the device? There is every reason to be optimistic, because of the broad importance of wireless to individuals and the economy in general, the widespread cooperation among industry and government in developing joint standards, and a strong interest by all parties to achieve global interoperability in the future. Can the software radio be trusted to replace the traditional radio operator? ABSOLUTELY YES, with a little hard work.

\section{REFERENCES}

${ }^{[1]}$ SDR Forum Web Site (www.Sdrforum.org), 2000

${ }^{[2]} \mathrm{J}$. Mitola III, "Technical Challenges in the Globalization of Software Radio", IEEE Communications Magazine, February 1999

[3] "SDR Forum: Architecture and Elements of Software Defined Radio Systems as Related to Standards", SDRF Technical Report 2.0, March 1999

${ }^{[4]}$ Joint Tactical Radio System (JTRS) Operational

Requirements Document (ORD), 23 March 1998

${ }^{[5]}$ Federal Communications Commission (FCC) Notice of Inquiry Regarding Software Defined Radios, ET Docket No. 00-47, 21 March 2000

${ }^{\left[{ }^{[6]}\right.}$ E. M. Noam, "Taking the Next Step Beyond Spectrum Auctions: Open Spectrum Access", Columbia Institute for Tele-Information, Columbia University, 10 October 1995

${ }^{[7]}$ W. Kennard, FCC Chairman, Address to CTIA Wireless 2000 Convention, "Wire Less Is More", February 28, 2000 